\title{
The Cost-Effectiveness of Pregabalin Versus Gabapentin for Peripheral Neuropathic Pain (pNeP) and Postherpetic Neuralgia (PHN) in China
}

Bruce C. M. Wang · Dongdong Liu · Wesley E. Furnback · Fan Bifa •

Peng Dong · Li Xie · Gregory F. Guzauskas · Sali Zhang

Received: January 5, 2016 / Published online: March 1, 2016

(C) The Author(s) 2016. This article is published with open access at Springerlink.com

\section{ABSTRACT}

Introduction: Postherpetic neuralgia $(\mathrm{PHN})$, a type of peripheral neuropathic pain ( $\mathrm{pNeP})$, is the most common complication of herpes zoster. The objective of this analysis was to determine the cost-effectiveness of pregabalin compared with gabapentin in $\mathrm{pNeP}$ and $\mathrm{PHN}$ in China.

Methods: We developed a China-localized 12-week simulation model to determine the cost-effectiveness of pregabalin compared to gabapentin in 1000 patients with $\mathrm{pNeP}$ and PHN. We utilized a questionnaire of Chinese key opinion leaders to estimate the pre-treatment distribution of pain scores for pNeP and PHN. Treatment outcomes for

Enhanced content To view enhanced content for this article go to http://www.medengine.com/Redeem/ 8844F0603330AAEA.

B. C. M. Wang (ه) · W. E. Furnback ·

G. F. Guzauskas

Elysia Group, LLC, New York, NY, USA

e-mail: bruce.wang@elysiagroup.com

D. Liu · P. Dong · L. Xie · S. Zhang

Pfizer Investment Co., Ltd, Beijing, China

F. Bifa

China-Japan Friendship Hospital, Beijing, China pregabalin and gabapentin were acquired from the published literature.

Results: Treatment with pregabalin lead to 12-week decreases in pain scores of $0.6(\mathrm{pNeP})$ and 0.7 (PHN) when compared to patients receiving gabapentin, at an incremental cost per additional day of mild/no pain of $\$ 45$. The difference in mean days of no or mild pain, moderate pain, and severe pain was $8.8,-5.7$, and -3.1 , when comparing pregabalin and gabapentin, respectively. Pregabalin had more mean days with a >30\% (7.71 days), $40 \%$ (8.97 days), and 50\% reduction (9.97 days) in pain when compared with gabapentin. In the pNeP scenario, pregabalin was associated with a lower average pain score compared with gabapentin (3.91 vs. 4.55). The difference in mean days of no or mild pain, moderate pain, and severe pain was $9.39,-5.56$, and -3.82 , when comparing pregabalin and gabapentin, respectively. Pregabalin had more mean days with a $>30 \%$ (8.77 days), 40\% (9.81 days), and $50 \%$ reduction (10.55 days) in pain when compared with gabapentin.

Conclusion: Pregabalin is an effective treatment for $\mathrm{PHN}$ and even for $\mathrm{pNeP}$ extensively, but at increased cost. It leads to 
improved outcomes including lower pain scores and more days with no or mild pain.

Funding: Pfizer, China.

Keywords: Cost-effectiveness; Gabapentin; Peripheral neuropathic pain (pNeP); Postherpetic neuralgia (PHN); Pregabalin

\section{INTRODUCTION}

Peripheral neuropathic pain ( $\mathrm{pNeP})$ is a general pain disorder caused by a lesion or disease of the peripheral somatosensory nervous system and occurs frequently with diabetes or following herpes zoster reactivation. The symptoms of pNeP may include numbness, tingling, weakness, and burning, and may lead to more severe events such as higher blood pressure, vomiting, and diarrhea. Postherpetic neuralgia (PHN) is a persistent, debilitating type of $\mathrm{pNeP}$ lasting months to years and is the most common complication of herpes zoster. $\mathrm{PHN} / \mathrm{pNeP}$ severity is a primary predictor of negative health impact, with the more severe patients reporting low levels of health-related quality of life [1].

China's rapidly aging population portends a coming transition from acute illness as its primary health challenge to chronic diseases [2], which require long-term, tailored solutions based on individual patient characteristics. It is generally believed that the prevalence of $\mathrm{pNeP}$ and PNP will continue to increase globally due to the aging of the population and higher survival rates from conditions that are associated with pNeP (such as cancer, HIV infection, and diabetes) [3].

Vera-Llonch et al. [4] developed a 12-week stochastic simulation model to examine the treatment of pNeP; this work was adapted by Tarride et al. [5] into a cost-effectiveness model comparing pregabalin versus gabapentin in the management of diabetic peripheral neuropathy (DPN) and PHN in a Canadian setting. Compared to gabapentin, pregabalin was projected to result in 6 and 9 additional days with no or mild pain, and 0.005 and 0.009 quality-adjusted life years (QALYs), for patients with DPN and PHN, respectively. The adapted Canadian model utilized a global perspective to facilitate future adaptations to the healthcare perspectives of additional countries.

Although the cost-effectiveness of pregabalin has previously been assessed in the UK [6] and Spain [7], no comparable studies to date have assessed the costs and health impacts for China. The objective of this study was to assess the cost-effectiveness of pregabalin compared with gabapentin in pNeP and PHN from a Chinese healthcare perspective using a China-localized adaptation of the Tarride et al. [5] model.

\section{METHODS}

\section{Approach}

We adapted the Tarride et al. [5] decision model to evaluate the short-term incremental cost-effectiveness of pregabalin versus gabapentin in China [8]. The analysis was conducted from a Chinese health system perspective using a 12-week time horizon based on duration of follow-up in the pregabalin clinical trials $[9,10]$. Treatment strategies included pregabalin and generic gabapentin, with the goal of treatment being to reduce daily pain severity. China-specific alterations to the Tarride et al. [5] model included resource utilization rates, resource costs, drug costs, treatment pathways, and mortality. Due to the short 12-week time horizon, we excluded costs and outcomes discounting, as well as consideration of QALYs 
and thus incremental cost-effectiveness ratios (ICERs). The decision to exclude the ICER also stems from a World Health Organization suggestion that gross domestic product-based willingness-to-pay per QALY thresholds in developing countries such as China are imperfect and should consider the relevant context of other available public health options, healthcare budgets, and the role of decision makers [11]. All costs are presented in 2015 US dollars and were converted using an exchange rate of $6.07 \mathrm{RMB}=1 \mathrm{USD}$. The model was programmed in Microsoft Excel (Microsoft Corporation, Redmond, WA, USA).

\section{Model Structure}

We utilized a three-state (severe, moderate, or no pain) Markov model (Fig. 1) to represent variable pain scores and associated health state utilities and costs, with a cycle length of 1 day. For any given patient, pain was assumed to vary daily, thus patients could move from one state to another with each cycle. Patient resource use was calculated using a decision analytic model (Fig. 2) based on the probability of a general practitioner visit followed by subsequent probabilities for additional resources.

\section{Model Outcomes}

The primary model outcomes were: (1) post-treatment pain score, (2) total number of days with no or mild pain, (3) drug costs, and (4) total medical-care services costs. Secondary outcomes included: (1) mean number of days with $30 \%, 40 \%$ and $50 \%$ reduction in pain scores, (2) mean number of days $\geq 2$ or 3 point reduction in pain, and (3) cost per additional day with no or mild pain. Pain was measured on an 11-point numeric pain rating scale $(0=$ no pain, $10=$ severe pain). Severe pain was classified as pain severity from 7 to 10 , moderate pain from 4 to 6 , and mild pain from 1 to 3 .

\section{Population}

We modeled the patient population based on clinical trial data of patients with $\mathrm{pNeP}$ which includes patients with both PHN and DPN, and/ or PHN. Patients had moderate to severe pain at treatment initiation, and received an average daily dose of $372 \mathrm{mg} /$ day for pregabalin and $2400 \mathrm{mg} /$ day for gabapentin, although dosage was modifiable through scenario analysis. Patient pain was assumed to be intractable to therapies for underlying conditions.

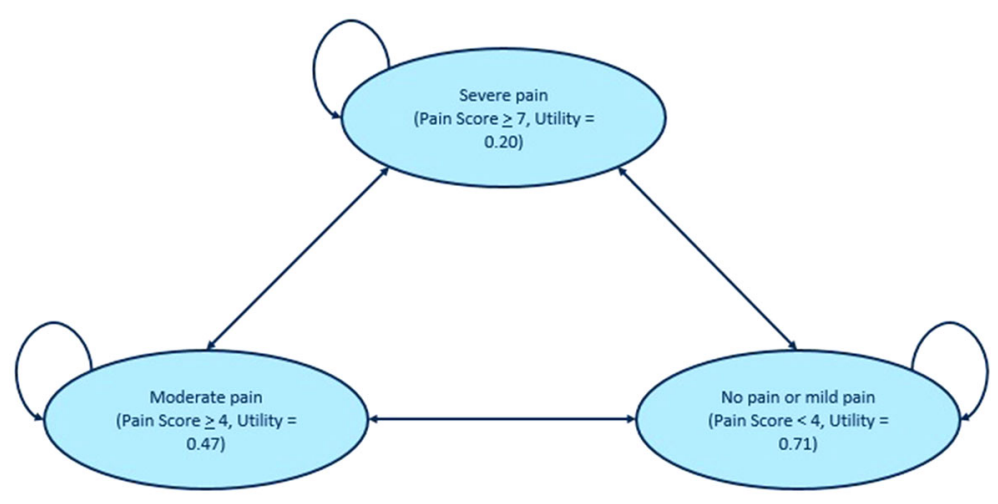

Fig. 1 Markov model structure. The Markov model structure consists of three health states: no pain or mild pain; moderate pain; and severe pain. Pain scores determine these health states, which each have individual utilities and probabilities of resource utilization and costs attached 


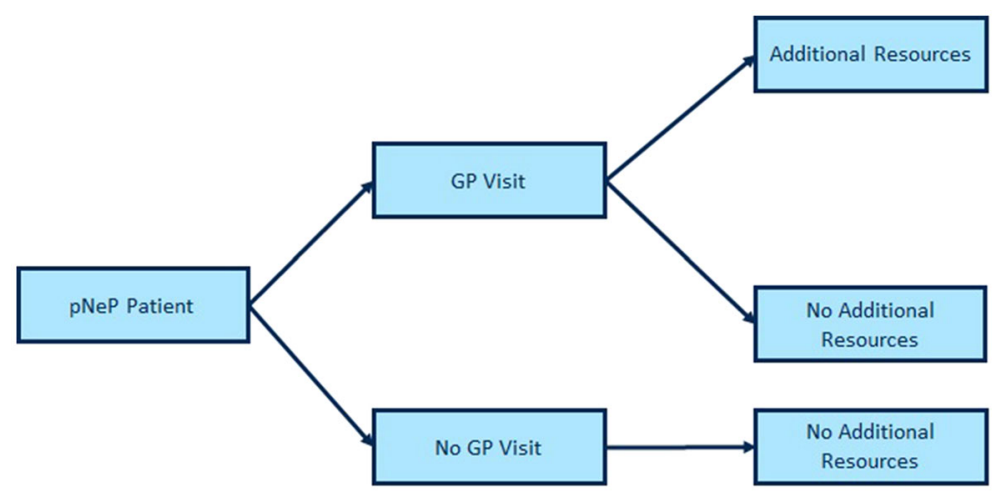

Fig. 2 Resource utilization decision tree. A probability of a doctor's visit is assigned to patients in each health state, patients then have probabilities of consuming additional resources and costs if they visit a doctor. GP general practitioner

\section{Model Parameters}

The model includes data from a variety of sources including physician surveys, randomized clinical trials, and published literature. We identified China-localized model parameters through literature review and a questionnaire of eleven key opinion leaders (Chinese specialists and general practitioners), who provided estimates of initial pain scores specific to the Chinese $\mathrm{pNeP}$ and $\mathrm{PHN}$ patient populations (Table 1). Efficacy estimates (Table 2) were obtained from published

Table 1 Pre-treatment pain scores

\begin{tabular}{llc}
\hline Score & pNeP (\%) & PHN (\%) \\
\hline 4.0 to $<5.0$ & 18.00 & 8.89 \\
5.0 to $<6.0$ & 18.18 & 15.33 \\
6.0 to $<7.0$ & 19.27 & 19.11 \\
7.0 to $<8.0$ & 19.55 & 18.89 \\
8.0 to $<9.0$ & 16.36 & 23.33 \\
9.0 to 10.0 & 8.64 & 14.44 \\
\hline
\end{tabular}

The pre-treatment pain score distributions were sourced from the physician survey. The distribution shows the percentage of patients within each pain score threshold for both the $\mathrm{pNeP}$ and $\mathrm{PHN}$ populations

$p N e P$ peripheral neuropathic pain, $P H N$ postherpetic neuralgia literature and were modeled for each week as the percentage reduction in baseline pain score [1]: pregabalin efficacy in $\mathrm{pNeP}$ was taken from Protocol 1008-155 [9] and has been used previously [4]; gabapentin efficacy in $\mathrm{pNeP}$ was taken from the pooled analysis of two randomized clinical trials $[12,13]$.

The probability of a doctor visit was unique to a patient's pain score on any given week and reflected results from the key opinion leader survey (Table 3). Patients who visited a doctor utilized healthcare resources according to results from the survey (Table 4). Drug costs included the cost of pregabalin and gabapentin (Table 5). The cost of pregabalin was based on the average trial dose of $372 \mathrm{mg}$ per day.

\section{Assumptions}

We assumed clinical management was similar for PHN and DPN patients. Adverse events were not included in the model because no clinically relevant differences exist between pregabalin and gabapentin within the 12-week time horizon. Rates of therapy discontinuation due to adverse events or lack of efficacy were also assumed to be the same between therapies. We assumed primary care providers clinically managed all patients, and that the likelihood 
Table 2 Weekly percentage reduction in pain score

\begin{tabular}{llllll}
\hline Week & \multicolumn{2}{l}{ Peripheral neuropathic pain } & & \multicolumn{2}{l}{ Postherpetic neuralgia } \\
\cline { 2 - 3 } & Pregabalin (\%) & Gabapentin $\mathbf{2 4 0 0 ~} \mathbf{~ m g ~ ( \% ) ~}$ & & Pregabalin (\%) & Gabapentin 2400 mg (\%) \\
\hline 1 & 13.7 & 17.2 & 14.7 & 17.3 \\
3 & 23.2 & 24.2 & 23.3 & 24.9 \\
3 & 29.9 & 27.5 & 29.5 & 29.2 \\
4 & 39.1 & 29.9 & 40.3 & 31.2 \\
5 & 44.4 & 31.5 & 46.6 & 32.7 \\
6 & 44.2 & 32.7 & 43.9 & 33.7 \\
7 & 45.0 & 33.6 & 43.9 & 34.5 \\
8 & 46.3 & 34.2 & 45.1 & 35.0 \\
9 & 49.8 & 34.6 & 49.1 & 35.4 \\
10 & 51.1 & 34.9 & 50.6 & 35.7 \\
11 & 53.3 & 35.2 & 57.5 & 35.9 \\
12 & 54.1 & 35.3 & 58.2 & 36.0 \\
\hline
\end{tabular}

The weekly percentage reductions in pain score are shown for each week of the model for both medications and populations. These percentages contribute to the transition probabilities within the Markov model

Table 3 Probability of a doctor visit according to physician survey

\begin{tabular}{lc}
\hline Health state & Probability (\%) \\
\hline Mild pain $(0.0$ to $<4.0)$ & 8.89 \\
Moderate pain $(4.0$ to $<7.0)$ & 15.33 \\
Severe pain $(7.0$ to $<10.0)$ & 19.11 \\
\hline
\end{tabular}

that patients will visit their primary care provider in a given week was a function of average level of pain. The efficacy of additional healthcare services is unknown, particularly if used in conjunction with pregabalin or other pharmacotherapy, thus we did not assign health benefits to them.

\section{Analysis}

The 12-week model was designed to estimate the impact of pharmacotherapy on daily pain experience using Bayesian simulation techniques to estimate patients' daily pain experience (measured on 0-10 scale) over time. The expected mean reductions (vs. baseline) in average daily pain scores for therapies of interest were applied to each patient's daily pain scores on a weekly basis to generate expected daily pain scores with treatment. We calculated various summary measures of patient outcomes based on expected daily pain scores for each treatment of interest. Summary measures of the outcomes were combined with utilization of healthcare services, costs of care, and health state utilities to calculate the cost-effectiveness of pregabalin versus gabapentin. We explored parameter uncertainty using a probabilistic sensitivity analysis of 1000 simulations of 1000 patients each, and calculated the mean and 95\% credible interval for each model outcome. 
Table 4 Resource utilization

\begin{tabular}{llll}
\hline Resource & Probability & Cost per unit & Number of units \\
\hline Doctor visit & See Table 3 & $\$ 52.72$ & 1 \\
Computed tomography (CT Scan) & 0.231 & $\$ 55.19$ & 1 \\
Magnetic resonance imaging (MRI) & 0.23222 & $\$ 131.14$ & 1 \\
Nerve conduction studies & 0.415 & $\$ 41.52$ & 1 \\
Quantitative sensory testing & 0.485 & $\$ 21.97$ & 1 \\
Doppler sonograph & 0.121111 & $\$ 27.18$ & 1 \\
Electromyography (EMG) & 0.44090 & $\$ 47.12$ & 1 \\
Transcutaneous electronic nerve stimulation & 0.16857 & $\$ 27.80$ & 1 \\
Implantation of spinal stimulator & 0.018 & $\$ 20.560$ & 1 \\
Physical therapy & 0.538888 & $\$ 27.41$ & 2 \\
Nerve block & 0.526666 & $\$ 135.42$ & 1.33333 \\
\hline
\end{tabular}

Resource utilization probabilities and costs were sourced from the physician survey. The probability of a doctor's visit was based on the patient's health state (Table 3). The probabilities of the other resources being utilized were assigned to patients who visited the doctor

Table 5 Drug costs

\begin{tabular}{lll}
\hline $\begin{array}{l}\text { Input (average } \\
\text { daily dose })\end{array}$ & $\begin{array}{l}\text { Cost per } \\
\text { day }\end{array}$ & Source \\
\hline $\begin{array}{l}\text { Pregabalin } \\
(372 \mathrm{mg} / \text { day })^{\mathrm{a}}\end{array}$ & $\$ 8.32$ & Pfizer, data on file \\
$\begin{array}{l}\text { Gabapentin } \\
(2400 \mathrm{mg} / \text { day })\end{array}$ & $\$ 2.96$ & Pfizer, data on file \\
\hline a & The average dose of pregabalin was $372 \mathrm{mg}$ per day
\end{tabular}

\section{Compliance with Ethics Guidelines}

This analysis in this article is based on previously conducted studies and does not involve any new studies of human or animal subjects performed by any of the authors.

\section{RESULTS}

The results of the pNeP and PHN analyses are summarized in Table 6. The mean pre-treatment pain scores were 6.7 for patients with pNeP and 7.2 for patients with PHN. At 12 weeks post-treatment initiation, the mean pain scores for the pNeP population were 3.9 and 4.5 for those treated with pregabalin and gabapentin, respectively. In the PHN population, 12-week mean pain scores were 4.2 and 4.9 for patients treated with pregabalin and gabapentin, respectively. Treatment with pregabalin resulted in an additional decrease of 0.6 and 0.7 when compared to patients receiving gabapentin in the $\mathrm{pNeP}$ and $\mathrm{PHN}$ populations, respectively.

In the $\mathrm{pNeP}$ population, pregabalin resulted in an increase of 9.5 days of no or mild pain, a decrease of -6.1 days of moderate pain, and a decrease of -3.5 days of severe pain compared to gabapentin. Pregabalin had more mean days with a $\geq 30 \%$ (8.3 days), 40\% (9.7 days), and $50 \%$ reduction (10.8 days) in pain when compared with gabapentin. In the PHN population, pregabalin resulted in an increase of 9.6 days of no or mild pain, a decrease of 
Table 6 Model outcomes

\begin{tabular}{|c|c|c|c|c|c|c|}
\hline \multirow[t]{2}{*}{ Outcomes } & \multicolumn{3}{|l|}{$\mathrm{pNeP}$} & \multicolumn{3}{|l|}{ PHN } \\
\hline & Pregabalin & Gabapentin & Difference & Pregabalin & Gabapentin & Difference \\
\hline $\begin{array}{c}\text { Pre-treatment } \\
\text { pain score }\end{array}$ & $6.7(0)$ & $6.7(0)$ & $0(0)$ & $7.2(0)$ & $7.2(0)$ & $0(0)$ \\
\hline $\begin{array}{l}\text { Post-treatment } \\
\text { pain score }\end{array}$ & $3.9(0.02)$ & $4.5(0.02)$ & $-0.6(0.03)$ & $4.2(0.02)$ & $4.9(0.02)$ & $-0.7(0.03)$ \\
\hline $\begin{array}{l}\text { Days no/mild } \\
\text { pain }\end{array}$ & $40.1(0.3)$ & $30.5(0.3)$ & $9.5(0.42)$ & $35.5(0.32)$ & $25.9(0.30)$ & $9.6(0.44)$ \\
\hline $\begin{array}{l}\text { Days moderate } \\
\text { pain }\end{array}$ & $30.2(0.3)$ & $36.3(0.32)$ & $-6.1(0.44)$ & $31.3(0.32)$ & $36.8(0.32)$ & $-5.5(0.46)$ \\
\hline $\begin{array}{c}\text { Days severe } \\
\text { pain }\end{array}$ & $13.7(0.23)$ & $17.2(0.25)$ & $-3.5(0.34)$ & $17.2(0.25)$ & $21.3(0.27)$ & $-4.1(0.37)$ \\
\hline $\begin{array}{c}\text { Days } \geq 30 \% \\
\text { reduction }\end{array}$ & $50.9(0.34)$ & $42.6(0.36)$ & $8.3(0.5)$ & $50.6(0.33)$ & $42.8(0.36)$ & $7.8(0.48)$ \\
\hline $\begin{array}{c}\text { Days } \geq 40 \% \\
\text { reduction }\end{array}$ & $44.4(0.34)$ & $34.7(0.35)$ & $9.7(0.47)$ & $43.3(0.34)$ & $34.1(0.35)$ & $9.2(0.47)$ \\
\hline $\begin{array}{c}\text { Days } \geq 50 \% \\
\text { reduction }\end{array}$ & $37.5(0.34)$ & $26.7(0.33)$ & $10.8(0.46)$ & $36.3(0.34)$ & $26.1(0.33)$ & $10.2(0.46)$ \\
\hline $\begin{array}{l}\text { Days } \geq 2 \text {-point } \\
\text { reduction }\end{array}$ & $56.2(0.32)$ & $49.3(0.36)$ & $6.9(0.49)$ & $57.4(0.33)$ & $51.2(0.35)$ & $6.2(0.48)$ \\
\hline $\begin{array}{l}\text { Days } \geq 3 \text {-point } \\
\text { reduction }\end{array}$ & $42.2(0.34)$ & $32.8(0.33)$ & $9.4(0.47)$ & $44.4(0.33)$ & $35.8(0.34)$ & $8.6(0.46)$ \\
\hline Drug costs & $\$ 685(\$ 0)$ & $\$ 243(\$ 0)$ & $\$ 441(\$ 0)$ & $\$ 685(\$ 0)$ & $\$ 243(\$ 0)$ & $\$ 441(\$ 0)$ \\
\hline Non-drug costs & $\$ 731(\$ 88.79)$ & $\$ 745(\$ 89.16)$ & $-\$ 15(\$ 121.86)$ & $\$ 742(\$ 89.75)$ & $\$ 755(\$ 88.27)$ & $-\$ 13(\$ 120.98)$ \\
\hline Total costs & $\$ 1415(\$ 88.81)$ & $\$ 989(\$ 89.36)$ & $\$ 427(\$ 122.27)$ & $\$ 1427(\$ 89.74)$ & $\$ 998(\$ 88.07)$ & $\$ 429(\$ 120.97)$ \\
\hline
\end{tabular}

The model outcomes for both the $\mathrm{pNeP}$ and PHN patient populations are presented as the mean (standard deviation) result of each simulation

$p N e P$ peripheral neuropathic pain, $P H N$ postherpetic neuralgia

-5.5 days of moderate pain, and a decrease of -4.1 days of severe pain compared to gabapentin. Pregabalin had more mean days with a $\geq 30 \%$ (7.8 days), $40 \%$ (9.2 days), and $50 \%$ reduction (10.2 days) in pain when compared with gabapentin.

The cost per additional day of mild/no pain was calculated for both patient populations
(Tables 7,8$)$. The total costs were higher in both populations in patients treated with pregabalin. In $\mathrm{pNeP}$ patient pregabalin resulted in an additional $\$ 427$ in total costs when compared with gabapentin. For the PHN population, total costs were an additional $\$ 429$ for pregabalin treatment compared to gabapentin. 
Table 7 Cost per additional day of mild/no pain

\begin{tabular}{lllr}
\hline Variable & Pregabalin & Gabapentin & Difference \\
\hline Days of mild/no pain & $40.06(39.46-40.67)$ & $30.52(29.90-31.11)$ & $9.53(8.74-10.34)$ \\
Total costs & $\$ 1415(\$ 1253-\$ 1599)$ & $\$ 989(\$ 821-\$ 1169)$ & $\$ 427(\$ 199-\$ 678)$ \\
Cost per day of mild/no pain & & & $\$ 44.74(\$ 20.96-\$ 71.91)$ \\
\hline
\end{tabular}

The results of the peripheral neuropathic pain population analysis are shown. The results are presented as the mean (95\% confidence interval) of the simulations

Table 8 Cost per additional day of mild/no pain

\begin{tabular}{lllr}
\hline Variable & Pregabalin & Gabapentin & Difference \\
\hline Days of mild/no pain & $35.50(34.86-36.12)$ & $25.91(25.35-26.50)$ & $9.59(8.71-10.44)$ \\
Total costs & $\$ 1427(\$ 1257-\$ 1612)$ & $\$ 998(\$ 830-\$ 1180)$ & $\$ 429(\$ 203-\$ 692)$ \\
Cost per day of mild/no pain & & & $\$ 44.69(\$ 20.92-\$ 71.91)$ \\
\hline
\end{tabular}

The results of the postherpetic neuralgia population analysis are shown. The results are presented as the mean (95\% confidence interval) of the simulations

In both populations, pregabalin treatment resulted in more additional days on mild/no pain and higher costs. In the pNeP patient population, treatment with pregabalin resulted in 9.5 additional days of mild/no pain at an additional cost of $\$ 427$, resulting in an incremental cost per additional day of mild/no pain of $\$ 44.69$. In the PHN patient population, treatment with pregabalin had 9.6 additional days of mild/no pain at $\$ 429$ additional cost, resulting in an incremental cost per additional day of mild/no pain of $\$ 44.74$.

\section{DISCUSSION}

We developed a China-localized decision model to determine the cost-effectiveness of pregabalin compared with gabapentin in $\mathrm{pNeP}$ and PHN patient populations. We found that treatment with pregabalin leads to decreases in pain scores of $0.6(\mathrm{pNeP})$ and $0.7(\mathrm{PHN})$ when compared to patients receiving gabapentin, at an incremental cost per additional day of mild/ no pain of $\$ 45$. Treatment with pregabalin provided 9.5 and 9.6 additional days of mild/ no pain and in the $\mathrm{pNeP}$ and $\mathrm{PHN}$ patient populations, respectively, suggesting that substantial improvements in patient quality of life are possible for these patient populations. Our results were robust to variation in model parameters in probabilistic sensitivity analysis.

pNeP represents a broad class of neuropathies associated with high pain levels, low utility scores, poor sleep, depression, and anxiety, though not on survival. Adding to this complexity, approximately half of $\mathrm{pNeP}$ patients are satisfied overall with their current pain medication [14]. Therefore, it is imperative to make more treatment options available to patients, so that a multifaceted approach to treatment is possible for each unique expression of patient pain. Given the increasing societal burden of these chronic diseases in China, government payers may need to increase coverage for effective treatments such as pregabalin. This will provide the necessary access to the medications that can improve 
quality of life and thus, potentially, increase productivity and output for the economy.

Randomized, placebo-controlled clinical trials have shown that pregabalin provides statistically and clinically significant improvements in pNeP associated with DPN and PHN [15-19]. The results of our modeling study are similar to the previous Canadian setting adaptation (6 and 9 additional days with no or mild pain for patients with DPN and PHN, respectively). However, our model differed from this previous model by excluding consideration of QALYs because of the perceived limitations imposed by the short time horizon of our model. Furthermore, there has been a shift toward alternative metrics of pNeP health outcomes because clinicians are increasingly appreciating outcomes reduction, such as clinical cure, days with no pain, and glycated hemoglobin (HbA1c), because of the ease of measurement and explanation to patients [20-22]. Lastly, we recognize that the QALY may not be the measure of choice for the Chinese health system, and that pain-free days are more likely to have buy-in from patients and clinicians alike.

Our model had several other limitations worth noting. First, we limited the time horizon to a 12-week period of follow-up based on available trial data; pain in patients with peripheral neuropathies may last for much longer. Second, we did not consider adverse events in our model because we assumed adverse event rates and rates of discontinuation due to adverse events were similar for both treatment populations, primarily because of the short time horizon chosen for the model. In a longer-term model, these rates would be expected to exhibit differences. Third, patients who were referred to specialists were assumed to have received additional healthcare services, which may have had an impact on the costs associated with any given treatment strategy.

Fourth, the model was primarily based on randomized clinical trial data of predominantly white patients that may not represent the real treatment effects, heterogeneity, and drug adherence in a Chinese setting. If China-specific clinical trial data were available we would have used it instead; nonetheless, clinical trial evidence is the gold standard of evidence for decision modeling studies, and while the populations studied may be highly specific, the data produced in the trials is of greater accuracy and credibility than educated guesses. The incorporation of data from other studies may provide different estimates than those we have calculated; however, we believe the parameters selected for this model represent the best available evidence. Lastly, we chose to omit the standard ICER measure of cost-effectiveness because the model's time horizon is brief at only 12 weeks, and because $\mathrm{pNeP} / \mathrm{PHN}$ is not a fatal condition, thus any assessment of survival and quality-adjusted survival is inherently limited. In lieu of an ICER, the cost per day of no/mild pain is a plain language representation of value that may be of greater use in the Chinese healthcare setting.

\section{CONCLUSIONS}

Pregabalin appears to be an effective treatment for PHN and pNeP at an incremental cost per additional day of mild/no pain of $\$ 45$, leading to improved outcomes including lower pain scores and an increase in days with no or mild pain, in a Chinese setting. Adding pregabalin to the treatment paradigm for $\mathrm{pNeP} / \mathrm{PHN}$ would provide physicians with an additional weapon to treat patients with $\mathrm{pNeP} / \mathrm{PHN}$ that provides a great value. 


\section{ACKNOWLEDGMENTS}

This study was funded by Pfizer, China. The article processing charges for this publication were funded by Pfizer, China. All named authors meet the International Committee of Medical Journal Editors (ICMJE) criteria for authorship for this manuscript, take responsibility for the integrity of the work as a whole, and have given final approval to the version to be published.

Disclosures. Bruce C. M. Wang is a paid consultant to Pfizer. Dongdong Liu is an employee of Pfizer. Wesley E. Furnback is a paid consultant to Pfizer. Fan Bifa acted as a consultant for Pfizer. Peng Dong is an employee of Pfizer. Li Xie is an employee of Pfizer. Gregory F. Guzauskas is a paid consultant to Pfizer. Sali Zhang is an employee of Pfizer.

Compliance with Ethics Guidelines. This analysis in this article is based on previously conducted studies and does not involve any new studies of human or animal subjects performed by any of the authors.

Open Access. This article is distributed under the terms of the Creative Commons Attribution-NonCommercial 4.0 International License (http://creativecommons.org/licenses/ by-nc/4.0/), which permits any noncommercial use, distribution, and reproduction in any medium, provided you give appropriate credit to the original author(s) and the source, provide a link to the Creative Commons license, and indicate if changes were made.

\section{REFERENCES}

1. Haythornthwaite JA, Benrud-Larson LM. Psychological aspects of neuropathic pain. Clin J Pain. 2000;16:S101-5.

2. Zhang Y, Feng Y, Qu Z, Qi Y, Zhan S. Current situation and challenge of registry in China. Front Med. 2014;8:294-9.

3. McDermott AM, Toelle TR, Rowbotham DJ, Schaefer CP, Dukes EM. The burden of neuropathic pain: results from a cross-sectional survey. Eur J Pain. 2006;10(2):127-35.

4. Vera-Llonch M, Dukes E, Delea TE, Wang ST, Oster G, Neuropathic Pain Outcomes Modeling Working Group. Treatment of peripheral neuropathic pain: a simulation model. Eur J Pain. 2006;10(3):279-85 [Epub 2005 Jun 23].

5. Tarride JE, Gordon A, Vera-Llonch M, Dukes E, Rousseau C. Cost-effectiveness of pregabalin for the management of neuropathic pain associated with diabetic peripheral neuropathy and postherpetic neuralgia: a Canadian perspective. Clin Ther. 2006;28(11):1922-34.

6. Gordon J, Lister S, Prettyjohns M, McEwan P, Tetlow A, Gabriel Z. A cost-utility study of the use of pregabalin in treatment-refractory neuropathic pain. J Med Econ. 2012;15(2):207-18.

7. Rodríguez MJ, Díaz S, Vera-Llonch M, Dukes E, Rejas J. Cost-effectiveness analysis of pregabalin versus gabapentin in the management of neuropathic pain due to diabetic polyneuropathy or post-herpetic neuralgia. Curr Med Res Opin. 2007;23(10):2585-96.

8. Wang BC, Liu D, Furnback W, Bifa F, Dong P, Xie L, Zhang S. Assessing the treatment of peripheral neuropathic pain (Pnep) and postherpetic neuralgia (Phn) in China using a simulation model. Value Health. 2015;18(7):A660.

9. Freynhagen R, Strojek K, Griesing T, Whalen E, Balkenohl M. Efficacy of pregabalin in neuropathic pain evaluated in a 12-week, randomised, double-blind, multicentre, placebo-controlled trial of flexible- and fixed-dose regimens. Pain. 2005;115:254-63. 
10. Rosenstock J, Tuchman M, LaMoreaux L, Sharma U. Pregabalin for the treatment of painful diabetic peripheral neuropathy: a double-blind, placebo-controlled trial. Pain. 2004;110:628-38.

11. Marseille E, Larson B, Kazi DS, Kahn JG, Rosen S. Thresholds for the cost-effectiveness of interventions: alternative approaches. Bull World Health Organ. 2015;93(2):118-24.

12. Backonja M, et al. Gabapentin for the symptomatic treatment of painful neuropathy in patients with diabetes mellitus. JAMA. 1998;280:1831-6.

13. Rowbotham M, Harden N, Stacey B, et al. for the Gabapentin Postherpetic Neuralgia Study Group. Gabapentin in the treatment of post-herpetic neuralgia: a randomized controlled trial. JAMA 1998;280:1837-42.

14. Tarride JE, Collet JP, Choiniere M, et al. The economic burden of neuropathic pain in Canada. J Med Econ. 2006;9:55-68.

15. Richter RW, Portenoy R, Sharma U, et al. Relief of painful diabetic peripheral neuropathy with pregabalin: a randomized, placebo-controlled trial. J Pain. 2005;6:253-60.

16. Lesser H, Sharma U, LaMoreaux L, Poole RM. Pregabalin relieves symptoms of painful diabetic neuropathy: a randomized controlled trial. Neurology. 2004;63:2104-10.

17. Sabatowski R, Galvez R, Cherry DA, et al. Pregabalin reduces pain and improves sleep and mood disturbances in patients with post-herpetic neuralgia: results of a randomised, placebo-controlled clinical trial. Pain. 2004;109:26-35.

18. Dworkin RH, Corbin AE, Young JP Jr, et al. Pregabalin for the treatment of postherpetic neuralgia: a randomized, placebo-controlled trial. Neurology. 2003;60:1274-83.

19. Freynhagen R, Strojek K, Griesing T, et al. Efficacy of pregabalin in neuropathic pain evaluated in a 12-week, randomised, double-blind, multi- centre, placebo-controlled trial of flexible- and fixed-dose regimens. Pain. 2005;115:254-63.

20. Wang B, Roth JA, Nguyen H, Felber E, Furnback W, Garrison LP. The short-term cost-effectiveness of once-daily liraglutide versus once-weekly exenatide for the treatment of type 2 diabetes mellitus in the United States. PLoS One. 2015;10(4):e0121915.

21. Chiou CF, Wang B, Caldwell R, Furnback W, Lee JS, Kothandaraman N, Wang J, Zhang F. The cost reduction in hospitalization associated with paliperidone palmitate in China, Korea, and Malaysia. Neuropsychiatr Dis Treat. 2015;11:1989-94.

22. Wang B, Fang CH, Furnback W, Kim R, Magyar A, Yang YW. Estimating the cost-effectiveness of linezolid for the treatment of methicillin-resistant Staphylococcus aureus (MRSA) nosocomial pneumonia in Taiwan. Barcelona: European Society of Clinical Microbiology and Infectious Diseases Meeting; 2014. 